\title{
Signing and the Brain: The Importance of Early Education for Deaf and Hard of Hearing Children
}

\author{
Jaimie Hong ${ }^{1}$, Rosemari $\mathrm{Nam}^{1}$ and Elizabeth DeMott ${ }^{1}$ \\ ${ }^{1}$ Metuchen High School, Metuchen, NJ, USA
}

\section{ABSTRACT}

Language skills are developed as one is exposed to auditory input from birth to adolescence. However, a majority of deaf and hard-of-hearing (DHH) individuals do not receive adequate—or appropriate—guidance from their parents at an early age. Since the brain develops significantly during the early years of childhood, children need robust and persistent instruction to develop the ability to recognize language. DHH children who lack the ability to perceive audio and spoken language, the universal language format, have poor outcomes in the future. In fact, hearing-disabled Americans are more likely to be unemployed, imprisoned, or have poor physical health. This paper will discuss the obstacles DHH people face in society as well as introduce a program to improve the standard of living for the deaf community. It will focus on preventative methods, most importantly, the adoption of a visual language, to protect DHH children from facing major neurological issues that would intrude on their ability to communicate with others fluently.

\section{Introduction - Current Challenges in the Deaf Community}

The deaf and hard-of-hearing (DHH) community faces a grim and distressing reality despite the major advances in language research and the amelioration of society's perception towards them. Worldwide, there are approximately 466 million people, adults and children included, who suffer from disabling hearing loss (World Health Organization, 2020). Despite this significantly large portion of impaired individuals, the DHH community still faces many shortcomings, reporting higher rates of unemployment in the U.S.: only $55.3 \%$ of deaf people are employed compared to $75.8 \%$ of hearing people (Lou Garberoglio et al., 2019). They also maintain a lower educational attainment status: $44.4 \%$ of the deaf population lacks a high school diploma compared to $18.7 \%$ of their hearing counterparts (Reilly \& Qi, 2011). These statistics may be intertwined with the fact that DHH people are more likely to be illiterate and even imprisoned (Humphries et al., 2008). What is the major factor responsible for these devastating statistics? The answer is brief yet all-encompassing: the lack of education and reliable guidance available for deaf and hard of hearing children.

According to the National Institute on Deafness and Other Communication Disorders, $90 \%$ of deaf children in the United States are born under hearing parents. However, only a minority of these parents actually provide an adequate learning environment for their children, with as many as $88 \%$ failing to promote American Sign Language to them (American Society for Deaf Children, 2019). This is due to the misconceptions prevalent among hearing parents about the "harms" of learning visual language, many believing that sign language usage would possibly stunt their child's ability to understand spoken language (National Association of the Deaf, 2014). Since DHH children are incapable of developing language through auditory input, they must learn a different form of communication that they can readily access such as sign language, but the negative perceptions underlying visual languages lead to language deprivation, the stunted development of cognitive systems responsible for language fluency and memory organization (Wilkinson \& Morford, 2020). Thus, many DHH children are left with a significantly reduced opportunity to become productive members of society.

Moreover, DHH individuals have one of the poorest health statuses in the United States. The DHH population displays higher rates of cardiovascular diseases, anxiety, and domestic violence (Barnett, 1999). They also have 
insufficient access to medical and health services (National Association of the Deaf, 2014). These conditions are the result of the language and cognitive barriers DHH individuals experience in their upbringing. DHH children who are not placed in a language-enriching environment never get to communicate with their doctors, and thus never learn the fundamentals of self and medical care. In addition, language deprivation in DHH individuals deteriorates abstract reasoning, a function necessary for people to engage in healthcare. A devastating result of this predicament is that DHH patients are "6.9 times more likely to have lower general health knowledge compared to hearing patients" (Wilkinson \& Morford, 2020, p. 1334). The lack of importance placed on DHH and visual language education from a young age leaves lasting effects on the livelihood of hearing-impaired individuals.

There is also a lack of specialized doctors available to offer thorough explanations on the negative implications of cochlear implant technology. As a result, up to $80 \%$ of deaf children in developed countries receive cochlear implants (CI) in hopes of improving their hearing. Parents of DHH children often believe that a CI will ensure that their children will be able to hear sufficiently and eventually grow accustomed to spoken and written language, eliminating the children's chance to learn a visual one. However, it is not clear whether cochlear implants ultimately help DHH children regain their hearing capabilities; successful cases are relatively minute (Wilkinson \& Morford, 2020). Moreover, with a price tag of $\$ 30 \mathrm{k}$ to $\$ 50 \mathrm{k}$, cochlear implants can actually do more harm than good. The spread of misinformation regarding CIs results in children becoming deprived of any form of language, unable to carry out basic cognitive functions in their later lives. Misconceptions about the reliability of CIs also result in parents not preparing rigorously for a deaf education, such as teaching their child sign language and consulting language professionals. The unfortunate consequence is that DHH children are not introduced to a valid language they can access. By the time a parent of a DHH child realizes that the CI is not providing sufficient audio input, it may be well past the critical language development point in the child's life.

\section{Cognitive Benefits of Signed Language}

In every human being, brain plasticity, or the ability of the brain to readily adapt to stimuli, is prominent in the first few years of the child's life (White, 2013). Without sufficient linguistic and communication input in early life, a child may never be capable of carrying out certain basic life functions. For example, limited access to language at a young age increases the risk of a child exhibiting language deprivation, which creates more learning obstacles in regular schooling and a higher degree of unemployment later in life (Chamberlain \& Mayberry, 2000).

In order to prevent language deprivation, DHH children need to be exposed to a language they can comprehend and take input from. However, they do not have the privilege of learning language through auditory awareness. Luckily, there is a simple solution to this problem: visual language. Contrary to common belief, sign language is a complex and meaningful way of communicating that can translate any sentence from spoken language and has the same linguistic properties as spoken languages (National Association of the Deaf, 2014). DHH children can adopt the same cognitive advancements required for future communication and higher-order thinking from sign language as a hearing individual would from a spoken one. The risk of facing language deprivation will be reduced dramatically, allowing them to develop the systems necessary to function in a society that bases its communication and advancements around a spoken language.

Early exposure to visual language facilitates a high level of language organization and promotes the cognitive growth required to read and write spoken language. It can possibly accelerate joint-attention capacities (when two people focus on the same thing), allowing children to self-regulate attention to visual languages, a trait that has been proven to contribute to the development of spoken language processing skills in children (Harris \& Chasin, 2005). These early visual skills correlate to a higher degree of reading and writing language development (Chamberlain \& Mayberry, 2000).

It is not necessary for children to understand the phonological structure of English in order to learn it. There are other efficient ways of teaching deaf children how to read, starting with ASL fluency. Mayberry et al.'s analysis in deaf students revealed that spoken phonological coding "only predicts about 10\% of reading outcome" (National 
Association of the Deaf, 2014, para. 9). They found that ASL fluency and print exposure were the largest contributors to the ability to read, proving that placing more importance on visual language exposure, rather than English language systems, would be more effective in setting deaf children up for literacy in their later lives.

It is also important to note that the early acquisition of sign language does not reduce a DHH child's motivation or capacity to learn a vocal language (Davidson et al., 2014). It is a vital step to avoiding language deprivation, thus opening the door to many more opportunities for language acquisition.

\section{The Importance of Bilingualism}

DHH children tend to lack proper language development, for the only way they can perceive an auditory language is by observing the movements of a speaking mouth. When DHH children lack exposure to a valid language that they can comprehend, they are less likely to be capable of understanding and utilizing language as they grow up (Wilkinson \& Morford, 2020).

A reliable method of ensuring a constant linguistic input is to use both signed and spoken language, introducing bilingualism in DHH children at an early age. Families that want their hearing-impaired children to develop spoken language skills cannot rely on the traditional methods used for language development in hearing children. Thus, to ensure that their children are not deprived of a mode of expression, they can enforce sign language education, along with written and spoken language exposure. In doing so, the DHH child will always have a medium through which they can communicate with others and avoid the cognitive shortcomings that result from a lack of exposure to language (Mayberry \& Lock, 2003).

In order for DHH children to become productive members of society, they need to be exposed to bilingualism. Learning both visual and spoken languages will ensure that a hearing-impaired child will never face language deprivation and will also help them properly integrate themselves into a society where vocal language trumps all other communication methods. Fluency in a language is most dependent on the amount of exposure an individual has in a given language, regardless of whether it is visual or spoken (Mayberry \& Lock, 2003). Therefore, it is very important that DHH children utilize both visual and spoken languages on a daily basis.

Many concerned parents of DHH children believe that learning both sign and spoken language at the same time will only confuse them, or even negatively impact their language development as a whole (Wilkinson \& Morford, 2020). However, these misconceptions have been repeatedly disproven through studies focusing on the cognitive development involved in learning both sign and spoken language. Bilinguals are not likely to be confused when utilizing their two native languages at the same time (Core et al., 2013) and are known to be aware of grammatical differences in their respective languages (Baker \& van den Bogaerde, 2008). The rate of vocabulary development in bilingual and monolingual children has no significant difference, and a Bialystok and Feng "study [identifies]... areas of earlier or faster development in bilinguals that arise as a result of being exposed to multiple language systems, such as...faster lexical development...and greater cognitive flexibility" (Wilkinson \& Morford, 2020, p.1331). The idea that learning two languages at once is harmful to a child's neurobiological development is entirely unfounded and inherently harmful to the well-being of the deaf community. Parents and concerned individuals alike need to realize that DHH children are capable of becoming bilingual, a tool that will pay off astronomically as they grow up.

\section{A Simple Solution: Back to the Basics}

Deaf and hard-of-hearing children need to be in an environment that will cultivate extensive language usage in order to prevent delays in education and development. Parents usually opt for cochlear implants, assuming that they will grant their children the ability to hear again and develop speech similar to normally-hearing children (Humphries et al., 2012). However, this ideology is nothing short of a misconception. In reality, the effectiveness of these high-cost implants is variable and unpredictable. Instead of investing time and resources into a device that can ultimately do 
more harm than good, we need to formulate a comprehensive and reliable program that will address the multiple barriers throughout the lives of the deaf or hard of hearing.

As stated above, the best solution for such children is to learn signed language from the earliest years of their development. Learning sign language is just as enriching as learning a written language, and it will set children up with the cognitive foundations necessary to later master spoken language (Humphries et al., 2012). We propose a theoretical program for parents or guardians of hearing-impaired children to enrich their children's lives with language and culture, while also ensuring that their disabilities will never stand in the way of their future.

\section{The Program}

In this educational program, we will focus on three major aspects of a child's life and explain how one can incorporate both sign language and spoken language in a hearing disabled child's environment from as early as six months old. It will also cover how to make an environment more accommodating to deaf individuals as a whole.

\section{1) Home}

The period between the ages of 0-2 is a critical time for language development in infants, and it is thus important that DHH children are exposed to as much language as early as possible (National Association of the Deaf, 2014). This can only be achieved if parents ensure that they are utilizing a language accessible to their children in their infant years.

Regardless of whether or not the parent or guardian of a DHH child decides to proceed with a CI, that child should always be exposed to a visual language as a preventative measure. Because CIs lack reliability, parents need a "safety net" to fall back on to ensure that their hearing-disabled child will always have the means of communicating from childhood to adulthood. From as early as six months (Thompson et al., 2007), parents can begin introducing simple visual signs to help their DHH children learn how to utilize language to communicate. Babies need to be cared for in unique ways, and visual signs can help them indicate what they need without having to use any audio. A parent can make a sign to represent words such as "bathroom" or "hungry" to not only provide a method of communication but also present the fundamentals of language to their DHH children. Through repetition and practice, their child will always be exposed to some form of language input.

Learning that a new-born child has an audio disability can be frightening and confusing for inexperienced parents. However, there are many programs available for families in similar situations that provide guidance in creating a safe and proactive environment for their children. For example, Hands and Voices is an online community of parents and professionals who provide support to families with DHH children (https://www.handsandvoices.org/) to advocate for better and more enriching environments for DHH children. An example of such a program is the Advocacy, Support, and Training (ASTra) Program, which guides DHH children's parents who are unaware of the educational and social environments their children need (Hands \& Voices). There is also the Guide By Your Side Program that appoints specially trained parents with DHH children to "work as 'Guides' directly with families who have just learned of their child's hearing condition" (Hands \& Voices, para. 1). Inexperienced parents can develop a parent-toparent connection with those who have faced similar situations in their families. These teaching experiences allow families to learn how to become educational advocates for their children, setting them up to eventually advocate for themselves.

Studies have revealed that a deaf child shows higher rates of success in mastering signed language when his or her whole family joins in by learning how to sign as well (Amador et al., 2019). Hearing people have the privilege of acquiring as much as $70-90 \%$ of their language development from "casual conversations" such as regular family discussions or storytelling (Wilkinson \& Morford, 2020). However, DHH individuals do not have this opportunity. If their families were to discuss with each other exclusively in a hearing language, they would not only feel isolated from 
others but also be deprived of vital cognitive development. As a family begins to communicate through a language in which each member can comprehend and take input, its hearing-impaired children become less likely to face language deprivation. There are many different websites and services that teach the basics of signing and communicating with it. Online classes from sites such as ASLMeredith, Sign It!, and SignOn are some popular choices for affordable lessons in sign language. In addition, family members who wish to become fluent in sign can take more in-depth courses at institutions such as Gallaudet University and American Sign Language University.

\section{2) School}

It is important to note that DHH children cannot solely rely on their parents or family members to expose them to language. DHH children of DHH parents who attended an ASL-instructed pre-school had a higher success rate in signing proficiency in comparison to those who did not (Amador et al., 2019). In addition, it has been reported that ASL fluency is greater in DHH children with DHH parents if they attend a school teaching in ASL before the age of 12 (Henner et al., 2016). Exposure at home is necessary for the prevention of language deprivation, but exposure at school is vital to ensure that the child has a solid foundation in language. The best choice would be for parents of DHH children to send their children to schools that offer both ASL instruction and English instruction, promoting bilingualism.

Bilingualism is the ideal language route for any hearing impaired individual, for it ensures that the individual is constantly being exposed to a language from a young age. It also ensures that he or she is able to communicate with the rest of the world with the use of spoken or written language. Once DHH children learn to communicate through sign language, they will also acquire the neurological foundations that will benefit them when learning a written language (Thompson, 2004). English can be taught to DHH individuals the same way that English is taught to those who learn English as a second language. It is important to note that a deaf child's educator should also be fluent in sign language, so that he or she can understand the obstacles in learning a language through a different medium (Thompson, 2004).

\section{3) Community}

For any individual, communication is key in ensuring a successful and enriching future. Therefore, another major factor that will further contribute to a DHH person's well-being is a sense of community. Networking will not only introduce deaf individuals to others who also understand the struggles of living in a language-speaking society, but it will also teach them how to function in society with respect to their deafness. Interpersonal relationships are vital to the mental health of any human being, and, thus, it is important for DHH individuals to have an environment where they can develop personal connections in the comfort of a language they can comprehend.

Parents of DHH children should seek communities focused on connecting those with hearing-disabilities. Such communities can help both the parents and their children find comfort in knowing that there are others who are going through the same obstacles and experiences. This valuable form of communication can improve the overall DHH experience in society and show deaf individuals that their disabilities do not define the opportunities they have in life. Some reliable communities include the American Society of Deaf Children, Beginnings For Parents of Children Who Are Deaf or Hard of Hearing, Inc., and BabyHearing.org. There, parents and children can create new relationships with others facing the same situation in their families. DHH children can connect with other children who have the same hearing-disabilities as they do, allowing them to create lasting interpersonal relationships with a diverse group of people, a key aspect for social development in children (Batten et al., 2014).

DHH children need to be able to communicate with health care workers and language professionals because if they are unable to do so, they will never understand the fundamentals and importance of self-care. One of the major causes of the DHH community's poor outcomes in the health care system is the lack of exposure to dialogue with their own doctors and specialists. Additionally, the lack of early language development prevents many of them from gaining 
the linguistic skills required to communicate with their doctors during check-ups. Therefore, there are two major steps parents should take in order to alleviate the unfortunate outcomes of the DHH community in the health care system. Firstly, as reinstated several times throughout this paper, parents of DHH children should expose their offspring to a visual language so they can develop the linguistic functions needed to communicate in medical facilities. Secondly, they should bring an interpreter, or act as one, when going to medical appointments with their DHH children to ensure that important information about their children's health status is relayed to the children themselves. Doing so will expose them to the fundamental ideas of health care, resulting in them taking better care of themselves later in their lives (Wilkinson \& Morford, 2020).

\section{Conclusion}

The DHH experience, as of right now, is disappointing. The reported higher rates of unemployment and low health statuses will never be alleviated if people fail to understand the reasons behind them. There is little to no effort in raising awareness about not only the grim outcomes of the DHH community but also the methods necessary to prevent these inadequacies. In short, when a child is deprived of a language from an infantile stage, he or she may never be able to communicate properly in society. This issue is undoubtedly heightened for deaf children, for they do not have a readily accessible language from birth and need to be provided with a different form of language to prevent linguistic deprivation. Parents and trained professionals alike need to understand that sign language is a valid human language that will provide children with the linguistic structures necessary to develop cognitive language systems.

By advocating for sign language exposure and early forms of communication for younger DHH children, we can transform the DHH experience and begin to appreciate alternate language forms as an art rather than as an inconvenience.

\section{Acknowledgments}

I would like to give an enormous thank you to Rosemari Nam for her indispensable guidance in my work. I would also like to thank Stacy Gil, for her contribution was paramount to the completion of this paper and guided me through my first taste of scientific research.

\section{References}

Amador, B., Morford, J.P., Wilkinson, E., \& A. Villwock. (2019, November). Predicting language proficiency of deaf children. Paper presented at the 44th Boston University Conference on Language Development, Boston University, Boston, MA.

Baker, A.E., \& van den Bogaerde, B. (2008). Sign bilingualism: Language development, interaction, and maintenance in sign language contact situations. John Benjamins Publishing. https://doi.org/10.1075/sibil.38.

Barnett, S. (1999). Clinical and cultural issues in caring for deaf people. Family Medicine, 31(1), 17-22.

Batten, G., Oakes P. M., \& Alexander T. (2014). Factors Associated With Social Interactions Between Deaf Children and Their Hearing Peers: A Systematic Literature Review. The Journal of Deaf Studies and Deaf Education, 19(3), 285-302. https://doi.org/10.1093/deafed/ent052 
Bialystok, E., \& Feng, X. (2009). Language proficiency and executive control in proactive interference: Evidence from monolingual and bilingual children and adults. Brain and Language, 109, 93-100.

https://doi.org/10.1016/j.bandl.2008.09.001.

Chamberlain, C., \& Mayberry, R. I. (2000). Theorizing about the relationship between ASL and reading. In C. Chamberlain, J. Morford, \& R. I. Mayberry (Eds.), Language Acquisition by Eye (pp. 221-259). Lawrence Erlbaum Associates.

Core, C., Hoff, E., Rumiche, R., \& Señor, M. (2013).Total and conceptual vocabulary in Spanish-English bilinguals from 22 to 30 months: implications for assessment. Journal of Speech, Language \& Hearing Research, 56(5), 16371649. https://doi.org/10.1044/1092-4388(2013/11-0044).

Davidson, K., Lillo-Martin, D., \& Chen Pichler, D. (2014). Spoken English language development among native signing children with cochlear implants. Journal of Deaf Studies and Deaf Education, 19(2), 238-250. https://doi.org/10.1093/deafed/ent045.

Deafness and hearing loss. (2020, March 1). World Health Organization. https://www.who.int/en/news-room/factsheets/detail/deafness-and-hearing-loss.

Harris, M., \& Chasin, J. (2005). Visual attention in deaf and hearing infants: the role of auditory cues. Journal of Child Psychology and Psychiatry, 46(10), 1116-1123. https://doi.org/10.1111/j.1469-7610.2005.00405.x.

Henner, J., Caldwell-Harris, C. L., Novogrodsky, R., \& Hoffmeister, R. (2016). American Sign Language syntax and analogical reasoning skills are influenced by early acquisition and age of entry to signing schools for the Deaf. Frontiers in Psychology, 7. https://doi.org/10.3389/fpsyg.2016.01982.

Humphries, T., Kushalnagar, P., Mathur, G., Napoli, D. J., Padden, C., Rathmann, C., \& Smith, S. R. (2012). Language acquisition for deaf children: Reducing the harms of zero tolerance to the use of alternative approaches. Harm Reduction Journal, 9. https://doi.org/10.1186/1477-7517-9-16.

Garberoglio, C. L., Cawthon, S., Palmer, L. J., \& Sales, A. (2019). Deaf People and Employment in the United States: 2019. U.S. Department of Education, Office of Special Education Programs, National Deaf Center on Postsecondary Outcomes. https://www.nationaldeafcenter.org/sites/default/files/Deaf\%20People\%20and\%20Employment $\% 20 \mathrm{in} \% 20$ the $\% 20$ United $\% 20$ States $\% 202019 \% 20(7.26 .19)($ ENGLISH)(WEB).pdf

Mayberry, R. I., \& Lock, E. (2003). Age constraints on first versus second language acquisition: evidence for linguistic plasticity and epigenesis. Brain and Language, 87(2), 369-383. https://doi.org/10.1016/S0093934X(03)00137-8.

Pick, L. (2013, November). Health care disparities in the deaf community. American Psychological Association. https://www.apa.org/pi/disability/resources/publications/newsletter/2013/11/deaf-community.

Thompson, J. (2004). Teaching English literacy to members of the deaf community: insights for bilingual programming [Master's thesis, Auckland University of Technology]. Auckland University of Technology Library. http://hdl.handle.net/10292/4811

Thompson, R. H., Cotnoir-Bichelman, N. M., McKerchar, P. M., Tate, T. L., \& Dancho, K. A. (2007). Enhancing early communication through infant sign training. Journal of Applied Behavior Analysis, 40(1), 15-23. https://doi.org/10.1901/jaba.2007.23-06. 
U.S. Department of Health and Human Services. (2019, March). American Sign Language. National Institute of Deafness and Other Communication Disorders. https://www.nidcd.nih.gov/health/american-sign-language\#: :text=American\%20Sign\%20Language $\% 20$ (ASL) $\% 20$ is, grammar $\% 20$ that $\% 20$ differs $\% 20$ from $\% 20$ English.\&text=It $\% 20$ is $\% 20$ the $\% 20$ primary $\% 201$ la nguage, many $\% 20$ hearing $\% 20$ people $\% 20$ as $\% 20$ well.

Stine, T. (2019, February 13). Why deaf children need ASL. American Society for Deaf Children. https://deafchildren.org/2019/02/why-deaf-children-need-asl/\#: :text=It\%20may\%20not\%20surprise $\% 20$ you,rest $\% 20$ of $\% 20$ their $\% 20$ child $\% 27 \mathrm{~s} \% 20$ life.

White, E. J., Hutka, S. A., Williams, L. J., \& Moreno, S. (2013). Learning, neural plasticity and sensitive periods: implications for language acquisition, music training and transfer across the lifespan. Frontiers in Systems Neuroscience, 7. https://doi.org/10.3389/fnsys.2013.00090.

Wilkens, C. P., \& Heir, T. P. (2008). Deaf education and bridging social capital: A theoretical approach. American Annals of the Deaf, 153(3), 275-284. https://doi.org/10.1353/aad.0.0050.

Wilkinson, E., \& Morford, J. P. (2020). How bilingualism contributes to healthy development in deaf children: A public health perspective. Maternal Health Journal, 24(11), 1330-1338. https://doi.org/10.1007/s10995-020-02976$\underline{6}$. 